\title{
Lipids as conductors in the orchestra of life
}

\author{
Christopher J.R. Loewen
}

\begin{abstract}
Address: Department of Cellular and Physiological Sciences, Life Sciences Institute, The University of British Columbia, 2350 Health Sciences Mall, Vancouver, British Columbia, Canada, V6T 1 Z3

Email: cloewen@mail.ubc.ca

FI000 Biology Reports 2012, 4:4 (doi:10.34I0/B4-4)

This is an open-access article distributed under the terms of the Creative Commons Attribution-Non Commercial License (http://creativecommons.org/licenses/by-nc/3.0/legalcode), which permits unrestricted use, distribution, and reproduction in any medium, provided the original work is properly cited. You may not use this work for commercial purposes.

The electronic version of this article is the complete one and can be found at: http://fl000.com/reports/b/4/4
\end{abstract}

\begin{abstract}
The lipid phosphatidic acid is an important metabolic intermediate in the biosynthesis of lipids in all eukaryotic cells, but it is even more than that. Phosphatidic acid is emerging as a lipid that is both composer and conductor, where in addition to its role as biosynthetic precursor (composer) it is also a potent signaling molecule (conductor) that integrates membrane biogenesis with nutrient sensing and cell growth. This article discusses recent advances in yeast that give praise for phosphatidic acid as one of life's conductors.
\end{abstract}

\section{Introduction}

Phospholipids are the core building blocks of most cellular membranes, without which cells themselves would not exist. The plasma membrane defines the outer-most boundary for the chemistry of life and the inner membrane systems or organelles provide an organisational framework to compartmentalise the chemical reactions that we know as cellular metabolism. And it is the combination of these activities, membrane biogenesis and energy metabolism, that enable cells to grow and multiply. It seems rather obvious then, that cells need to co-ordinate membrane biogenesis with basic metabolism to ensure successful replication and the passing-on of their genes. This is especially true for single-celled organisms that are, by nature, at the mercy of the elements and must respond to environmental changes quickly and efficiently, but is also tremendously important during embryo-genesis in metazoans, in which a single cell gives rise to a complex multicellular organism.

How then do cells co-ordinate membrane production with metabolism? To begin to answer this question we will turn to a simple model eukaryotic cell, the budding yeast Saccharomyces cerevisiae. Yeast, because it is singlecelled, has evolved elaborate and dynamic ways to control membrane synthesis in response to its environment. In the past three decades, tremendous progress has been made in defining the fundamental metabolic pathways that contribute to phospholipid synthesis and turnover as well as defining many of the regulatory mechanisms. Much of this foundation has arisen from genetic and biochemical characterisation of yeast mutants from a small group of key labs (for extensive reviews see [1-3]). Their work has paved the way for recent studies that, in large part through the use of new cell and systems biology approaches, are uncovering surprising links between membrane biogenesis and the cell cycle.

This article will discuss the pivotal role for the lipid phosphatidic acid as a signaling hub for the integration of metabolic signals with membrane biogenesis. But why phosphatidic acid? The reason is that phosphatidic acid is a key metabolic precursor in the synthesis of both phospholipids (which make up membranes) and neutral lipids (such as triacylglycerol that is stored in cytoplasmic lipid droplets) and therefore sits at the crossroads between membrane biogenesis and lipid storage (see Figure 1). What better a lipid to sense the levels of then? We will discuss the effects of key nutrients - inositol, zinc and glucose - on regulation of lipid metabolism, emphasising how these affect the signaling activity of phosphatidic acid to couple membrane biogenesis to metabolism. 
Figure I. Coupling membrane biogenesis to metabolism and the cell cycle

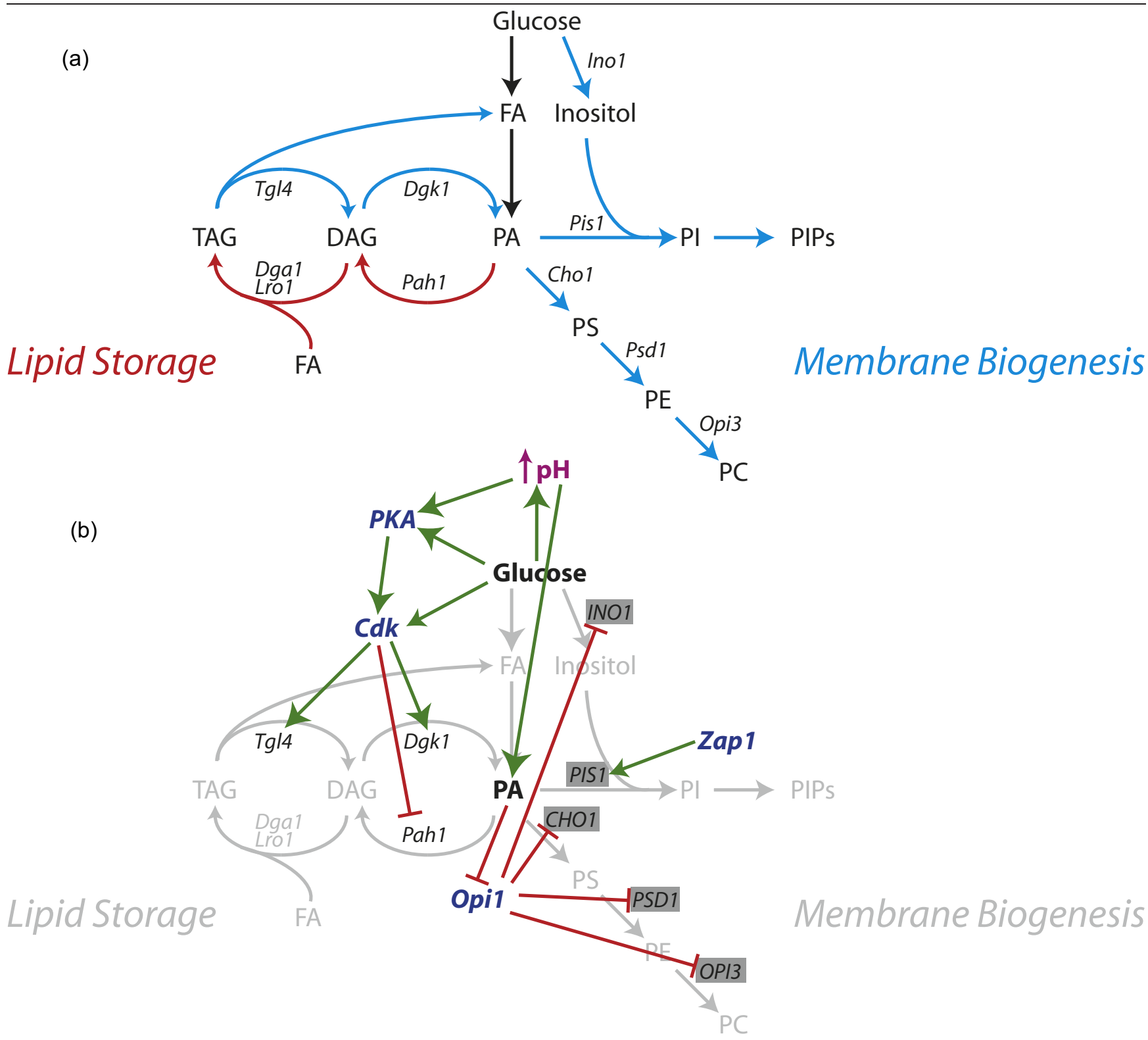

(a) Flow of metabolites between pathways that favour membrane biogenesis (blue) versus lipid storage (red). Arrows indicate metabolic steps, which are not necessarily direct. Key enzymes are shown in italics. (b) Regulation of lipid metabolism. Green arrows indicate activating steps while flattened red arrows indicate repressing steps. Regulatory factors are labelled in blue. Targets of transcriptional regulation are depicted in grey boxes. Activation of physiological cytosolic $\mathrm{pH}$ by glucose is depicted in purple. Not all depicted regulatory steps are direct and in some cases the specific molecular mechanisms are unknown. Abbreviations: Cdk, cyclin-dependent kinase; DAG, diacylglycerol; PA, phosphatidic acid; PI, phosphatidylinositol; PIP, phosphoinositides; PKA, protein kinase A; TAG, triacylglycerol.

\section{Control of membrane biogenesis, metabolism and the cell cycle}

Co-ordinate regulation of phospholipid metabolism by phosphatidic acid and the transcriptional repressor Opil Lipid metabolism is co-ordinately regulated at the transcriptional level by a yeast transcription factor called
Opi1, which binds to and represses the bHLH transcriptional activator complex Ino2-Ino4 at the promoters of over 30 phospholipid metabolic genes. This regulation has been extensively reviewed elsewhere [1]. Opi1 is repressed by binding directly to phosphatidic acid in the endoplasmic reticulum, which enables retention of Opi1 
outside of the nucleus away from the Ino2-Ino4 activator complex [4]. Opi1 specifically senses endoplasmic reticulum phosphatidic acid, because it is this pool of phosphatidic acid that acts as the metabolic precursor for de novo synthesis of phospholipids and neutral lipids. Depletion of endoplasmic reticulum phosphatidic acid causes rapid unbinding and translocation of Opi1 to the nucleus where it represses Ino2-Ino4 and the synthesis of phospholipids. Co-ordinate transcriptional regulation by Opi1 enables simultaneous control of synthesis of multiple classes of phospholipids. By inhibiting Opi1, phosphatidic acid promotes membrane biogenesis.

\section{Regulation by inositol}

Inositol is a critical precursor for the synthesis of phosphatidylinositol and the phosphoinositides, which are major components of yeast cell membranes. Inositol is a potent regulator of Opi1; it acts by depleting phosphatidic acid in the endoplasmic reticulum through its conversion into phosphatidylinositol by the action of phosphatidylinositol synthase (encoded by PIS1; see Figure 1). The gene most dynamically regulated by Opi1 is INO1, which encodes the rate-limiting enzyme in the synthesis of inositol from glucose. This provides tight feedback regulation of synthesis of inositol (i.e., high levels of inositol deplete phosphatidic acid, activating Opi1), thus conserving glucose in the presence of exogenous inositol. So why does the inositol signal act through phosphatidic acid and repress synthesis of other classes of phospholipids, namely phosphatidylcholine? One answer is that, in addition to conserving glucose for other metabolism, when inositol is abundant, yeast can build membranes primarily with phosphatidylinositol and therefore can afford to synthesize less phosphatidylcholine $[4,5]$. Abundance of inositol, likely from dead/ lysed yeast, may also indicate that other lipid precursors are abundant, such as choline/lyso-phosphatidylcholine that can be used to make phosphatidylcholine via salvage pathways [6], thus supporting cell growth and conserving glucose for other pathways. Thus, although it seems counterproductive that in the presence of inositol, de novo phospholipid synthesis is repressed, yeast are actually conserving energy by directing glucose away from inositol synthesis while simultaneously building membranes with phosphatidylinositol. This decision in the end supports membrane biogenesis and cell growth.

\section{Regulation by zinc}

Zinc is an essential mineral required for growth, where it acts as a cofactor for many metabolic enzymes. Zinc depletion can therefore generally be thought of as a stopgrowth signal and, in yeast, zinc depletion provides another example of co-ordinate regulation via phosphatidic acid signaling [1]. When cells are starved of zinc, the zinc-sensitive transcriptional activator Zap1 upregulates expression of two critical genes controlling the level of phosphatidic acid in the endoplasmic reticulum. Upregulation of phosphatidylinositol synthase, encoded by PIS1, lowers the level of endoplasmic reticulum phosphatidic acid through its conversion into phosphatidylinositol, while upregulation of phosphatidic acid phosphatase, encoded by PAH1, lowers endoplasmic reticulum phosphatidic acid through its conversion to diacylglycerol. This combined effect of lowering endoplasmic reticulum phosphatidic acid in response to zinc depletion results in co-ordinate repression of phospholipid metabolic genes by Opi1, which shuts-down de novo phospholipid synthesis. Regulation by zinc therefore provides the cell with a rapid mechanism to downregulate membrane biogenesis in anticipation of halted growth resulting from an eventual decrease in metabolism.

\section{Glucose, phosphatidic acid and the cell cycle}

Clearly membrane biogenesis must be coupled with the cell cycle such that the morphogenic cycle is in sync with replication of the nucleus. Glucose is in prime position as the master regulator of both the morphogenic and nuclear cycles, given its dual roles as metabolic precursor and energy source (see Figure 1). Glucose regulation of yeast growth has been reviewed extensively elsewhere [7]. Glucose regulates the nuclear cycle by activating the major Cdk in yeast, Cdc28, through a combination of mechanisms including activation of the cyclins [8], protein kinase A [9], TOR kinase [9] and likely through other as-yet undefined pathways. Yeast happily use glucose as their sole carbon source, meaning it is the precursor in the synthesis of all classes of lipids. But does the cell also use glucose availability as a signal regulating lipid metabolism?

Kurat et al. in a very lucid study have uncovered a direct link between the cell cycle and mobilisation of fat in the form of triacylglycerol [10]. They find that the activity of the triacylglycerol lipase, $\mathrm{Tgl} 4$, is directly regulated by phosphorylation by Cdc28. Cdk activation of $\mathrm{Tgl} 4$ coincides with and is required for cells to enter G1 of the cell cycle, and they find that the top signal for activation is glucose availability. Triacylglycerol hydrolysis in the presence of glucose mobilises fat stores into diacylglycerol and, through the co-operative action of diacylglycerol kinase (Dgk1) [11], into phosphatidic acid, which can then be used as precursors for the synthesis of phospholipids. The source of the triacylglycerol is likely cytoplasmic lipid droplets, since this is the location of the lipases, and lipid droplets are rapidly consumed upon glucose addition. In fact, when de novo fatty acid synthesis is blocked pharmacologically, lipid droplets are sufficient to provide the precursors for 
membrane synthesis and re-entry into the cell cycle [10]. An important consequence of this burst in triacylglycerol hydrolysis is an increase in phosphatidic acid in the endoplasmic reticulum, which, by repressing Opi1, turns on de novo phospholipid biosynthetic pathways. Thus, the signalling function of phosphatidic acid co-ordinates cell cycle-activated hydrolysis of fat with production of membranes, promoting cell growth in response to glucose.

If triacylglycerol hydrolysis is the yin of fat metabolism then phosphatidic acid hydrolysis is the yang. Pah1 is the major phosphatidic acid hydrolase responsible for conversion of phosphatidic acid in the endoplasmic reticulum into diacylglycerol $[12,13]$, which can then be used for the synthesis of triacylglycerol in lipid droplets [14]. Activation of Pah1 is therefore a key decision point for the cell since Pah1 acts on the pool of phosphatidic acid that is also the precursor for phospholipid synthesis and hence membrane biogenesis. In fact, loss of Pah1 prevents lipid droplet formation and decreases cellular triacylglycerol levels [14] and at the same time causes expansion of the endoplasmic reticulum [13]. Blocking de novo phospholipid synthesis, on the other hand, elevates phosphatidic acid levels in the endoplasmic reticulum, which results in increased triacylglycerol synthesis and the formation of so-called "super-sized" lipid droplets [15]. Phosphatidic acid may even have a regulatory role here beyond simply being a triacylglycerol precursor, where its presence on the surface of lipid droplets may control their size, number and fusion [15]. Importantly, balancing the flow of phosphatidic acid between neutral lipids and phospholipids is critical for cell growth and is an important function of Pah1.

Not surprisingly then, the activity of Pah1 is cell cycleregulated. Pah1 is phosphorylated by Cdk, which inhibits its hydrolase activity $[13,16]$. During periods of the cell cycle requiring membrane biogenesis, Pah1 is predominantly phosphorylated, which inhibits its activity, diverting phosphatidic acid away from neutral lipid synthesis and making it available for synthesis of phospholipids. Because this phosphatidic acid is located in the endoplasmic reticulum, it also serves a signalling function by regulating Opi1. The resulting increase in endoplasmic reticulum phosphatidic acid represses Opi1, which increases expression of phospholipid metabolic genes that further promotes phospholipid synthesis. Pah1 is dephosphorylated and positively regulated by a conserved phosphatase, Nem1; however, the links between Nem 1 and the cell cycle have yet to be established $[17,18]$. Nevertheless, constitutive activation of Pah1 through overexpression of Nem 1 arrests the cell cycle, which is likely a result of triacylglycerol synthesis at the expense of phospholipids, emphasising the impact that Pah1 and ultimately phosphatidic acid in the endoplasmic reticulum play in co-ordinating membrane biogenesis with growth.

\section{Phosphatidic acid is a $\mathrm{pH}$ biosensor}

An unanticipated recent discovery was a role for intracellular $\mathrm{pH}\left(\mathrm{pH}_{\mathrm{i}}\right)$ in metabolic control of lipid metabolism. pHi in all cells is rigorously maintained; however, until now it was unclear if $\mathrm{pH}_{\mathrm{i}}$ was also used as a biological signal. The major regulators of $\mathrm{pH}_{\mathrm{i}}$ in yeast are a P-type ATPase of the plasma membrane, Pma1, and the V-ATPase (for an excellent review see [19]). Pma1 controls $\mathrm{pH}_{\mathrm{i}}$ by pumping protons generated by glycolysis directly out of the cell [20], while the V-ATPase affects pHi directly and indirectly, by pumping protons from the cytoplasm into the vacuole and by regulating the trafficking of Pma1 [21]. Not surprisingly, glucose is also an important activator of the pumps, which prevents over-acidification of the cytoplasm as a consequence of glycolysis. Glucose starvation results in rapid pump inactivation and an acute drop in $\mathrm{pH}_{\mathrm{i}}$ from $\sim 7$ to $\sim 6$. However, it was never established if the drop in $\mathrm{pH}_{\mathrm{i}}$ was also a productive signal.

By taking a comprehensive systems-biology approach to uncover new regulators of phospholipid metabolism, Young et al. [22] have discovered that $\mathrm{pH}_{\mathrm{i}}$ is a signal regulating binding of phosphatidic acid to Opi1. Their genome-wide screen for yeast mutants that failed to grow in the absence of inositol found an enrichment for genes with functions in $\mathrm{pH}_{\mathrm{i}}$ regulation, including both the V-ATPase and Pma1. Acidification of the cytoplasm causes Opi1 to unbind the endoplasmic reticulum and translocate to the nucleus, turning off phospholipid metabolism. Because the headgroup of phosphatidic acid is a phosphomonoester (most other phospholipids have phosphodiesters) it has a $\mathrm{p} K_{\mathrm{a}}$ in the physiological range, which means it becomes protonated with decreasing $\mathrm{pH}_{\mathrm{i}}$ [23]. Young et al. find that Opi1 has lower affinity for protonated compared with deprotonated phosphatidic acid, and that binding is $\mathrm{pH}$-dependent. These results are consistent with the "electrostatic/hydrogen bond switch model", which provides an elegant explanation for charge-dependent phosphatidic acid binding [24]. Lastly, Young et al. [22] find that the drop in $\mathrm{pH}_{\mathrm{i}}$ upon glucose starvation is the physiological signal that releases Opi1 from phosphatidic acid in the endoplasmic reticulum.

Thus, in addition to its role as a metabolic precursor, glucose also exploits the signaling function of phosphatidic acid through changes in $\mathrm{pH}_{\mathrm{i}}$ to control lipid metabolism. In this special case, phosphatidic acid acts as a pH biosensor that detects the metabolic status of the cell and couples nutrient availability with membrane 
biogenesis. pH-sensing by phosphatidic acid enables the cell to rapidly shut down phospholipid synthesis in anticipation of depleted lipid precursors. But does $\mathrm{pHi}$ then also signal lipid storage? Work by Dechant et al. suggests it does $[25,26]$. They show that the drop in $\mathrm{pH}_{\mathrm{i}}$ upon glucose starvation is a signal regulating protein kinase A, which is likely sensed by the V-ATPase. Repression of protein kinase $\mathrm{A}$ by low $\mathrm{pH}_{\mathrm{i}}$ may divert phosphatidic acid away from phospholipids into neutral lipids by dampening Cdk (Cdc28) activity and lowering the activity of Tgl4 and Dgk1 while at the same time derepressing Pah1. Thus, co-ordinated signaling functions for $\mathrm{pH}_{\mathrm{i}}$, through both protein kinase A and phosphatidic acid directly, may be a means to rapidly balance the metabolic flux between lipid storage and membrane biogenesis.

\section{Conclusion}

Because of our recent comprehensive understanding of the lipid metabolic pathways, the location of the enzymes, and even more recently, of the diversity and location of the lipid species themselves (largely thanks to mass spectrometry technologies) yeast lipids research is poised to be at the forefront of new breakthroughs in lipid physiology. What does the future hold? Let us turn back to the crossroads, to phosphatidic acid, and to the cell's decision to store fat or to build membranes. Our work in yeast is telling us that phosphatidic acid is poised to be a key molecule regulating fat storage in humans, a process that is directly implicated in multiple metabolic diseases including obesity, type 2 diabetes and the lipodystrophies [27]. The obvious role for phosphatidic acid is as a lipid precursor, or composer, in the synthesis of triacylglycerol, a major constituent of lipid droplets. The availability of phosphatidic acid in the endoplasmic reticulum and the regulation of key enzymes, especially the lipin family of phosphatidic acid phosphatases, will be critical factors [27]. But what about phosphatidic acid as conductor? Tellingly, lipin plays a dual transcriptional role in regulating hepatic lipid homeostasis, which is now found to involve nutrient sensing by mTOR [28]. Phosphatidic acid is an important regulator of mTOR [29], suggesting that phosphatidic acid is also playing a signaling role in regulating lipid homeostasis in humans. It therefore seems that real parallels can be drawn between the yeast and mammalian systems, and that perhaps lipids, as conductors, are the key to co-ordinating membrane biogenesis with metabolism.

\section{Abbreviations}

bHLH, basic helix-loop-helix; Cdk, cyclin-dependent kinase; FA, fatty acid; mTOR, mammalian TOR; PC, phosphatidylcholine; $\mathrm{PE}$, phosphatidylethanolamine; $\mathrm{pHi}$, intracellular $\mathrm{pH}$; $\mathrm{PS}$, phosphatidylserine; TOR, target-of-rapamycin; V-ATPase, vacuolar ATPase.

\section{Competing interests}

The author declares that he has no competing interests.

\section{Acknowledgements}

The author thanks his lab, especially J. Shin and B. Young for critical discussions on the manuscript.

\section{References}

I. Carman GM, Han GS: Regulation of Phospholipid Synthesis in the Yeast Saccharomyces cerevisiae. Annu Rev Biochem 20I I, 80:859-83.

2. Gaspar ML, Aregullin MA, Jesch SA, Nunez LR, Villa-Garcia M, Henry SA: The emergence of yeast lipidomics. Biochim Biophys Acta 2007, I771:241-54.

3. Kohlwein SD: Triacylglycerol homeostasis: insights from yeast. J Biol Chem 2010, 285:I5663-7.

4. Loewen CJ, Gaspar ML, Jesch SA, Delon C, Ktistakis NT, Henry SA, Levine TP: Phospholipid metabolism regulated by a transcription factor sensing phosphatidic acid. Science 2004, 304: I644-7.

FI000 Factor 14

Evaluated by Antonella De Matteis 25 Jun 2004, Christoph Benning 09 Jul 2004, David K Banfield 27 Aug 2004

5. Ejsing CS, Sampaio JL, Surendranath V, Duchoslav E, Ekroos K, Klemm RW, Simons K, Shevchenko A: Global analysis of the yeast lipidome by quantitative shotgun mass spectrometry. Proc Natl Acad Sci U S A 2009, I06:2136-4I.

6. Riekhof WR, Wu J, Gijon MA, Zarini S, Murphy RC, Voelker DR: Lysophosphatidylcholine metabolism in Saccharomyces cerevisiae: the role of P-type ATPases in transport and a broad specificity acyltransferase in acylation. J Biol Chem 2007, 282:36853-6I.

FI000 Factor 6

Evaluated by Christopher Loewen 17 Jan 2012

7. Santangelo GM: Glucose signaling in Saccharomyces cerevisiae. Microbiol Mol Biol Rev 2006, 70:253-82.

8. Newcomb LL, Diderich JA, Slattery MG, Heideman W: Glucose regulation of Saccharomyces cerevisiae cell cycle genes. Eukaryot Cell 2003, 2:143-9.

FI000 Factor 8

Evaluated by Christopher Loewen 17 Jan 2012

9. Slattery MG, Liko D, Heideman W: Protein kinase A, TOR, and glucose transport control the response to nutrient repletion in Saccharomyces cerevisiae. Eukaryot Cell 2008, 7:358-67.

FI000 Factor 6

Evaluated by Christopher Loewen 17 Jan 2012

10. Kurat CF, Wolinski H, Petschnigg J, Kaluarachchi S, Andrews B, Natter K, Kohlwein SD: CdkI/Cdc28-dependent activation of the major triacylglycerol lipase Tgl4 in yeast links lipolysis to cell-cycle progression. Mol Cell 2009, 33:53-63.

FI000 Factor 10

Evaluated by Ruth Collins 30 Sep 2009

II. Fakas S, Konstantinou C, Carman GM: DGKI-encoded diacylglycerol kinase activity is required for phospholipid synthesis during growth resumption from stationary phase in Saccharomyces cerevisiae. J Biol Chem 20II, 286:I464-74.

FI000 Factor 6

Evaluated by Christopher Loewen 17 Jan 2012

12. Han GS, Wu WI, Carman GM: The Saccharomyces cerevisiae Lipin homolog is a Mg2+-dependent phosphatidate phosphatase enzyme. J Biol Chem 2006, 281:9210-8.

FI000 Factor 7

Evaluated by Timothy Durrett and John Ohlrogge 08 Sep 2009, Christopher Loewen 17 Jan 2012 
13. Santos-Rosa H, Leung J, Grimsey N, Peak-Chew S, Siniossoglou S: The yeast lipin Smp2 couples phospholipid biosynthesis to nuclear membrane growth. EMBO J 2005, 24:193I-4I.

FI000 Factor 6

Evaluated by Christopher Loewen 17 Jan 2012

14. Adeyo O, Horn PJ, Lee S, Binns DD, Chandrahas A, Chapman KD, Goodman JM: The yeast lipin orthologue $\mathbf{P a h} \mathbf{~ p}$ is important for biogenesis of lipid droplets. J Cell Biol 201 I, I 92: I043-55.

FI000 Factor 6

Evaluated by Christopher Loewen 17 Jan 2012

15. Fei W, Shui G, Zhang Y, Krahmer N, Ferguson C, Kapterian TS, Lin RC, Dawes IW, Brown AJ, Li P, Huang X, Parton RG, Wenk MR, Walther TC, Yang H: A role for phosphatidic Acid in the formation of "supersized" lipid droplets. PLoS Genet 201 I, 7: el002201.

FI000 Factor 6

Evaluated by Christopher Loewen 17 Jan 2012

16. O'Hara L, Han GS, Peak-Chew S, Grimsey N, Carman GM, Siniossoglou S: Control of phospholipid synthesis by phosphorylation of the yeast lipin PahIp/Smp2p Mg2+-dependent phosphatidate phosphatase. J Biol Chem 2006, 28I: 34537-48.

FI000 Factor 6

Evaluated by Christopher Loewen 17 Jan 2012

17. Kim Y, Gentry MS, Harris TE, Wiley SE, Lawrence JC, Jr., Dixon JE: A conserved phosphatase cascade that regulates nuclear membrane biogenesis. Proc Natl Acad Sci U S A 2007, 104: 6596-60I.

FI000 Factor 6

Evaluated by Christopher Loewen 17 Jan 2012

18. Siniossoglou S, Santos-Rosa H, Rappsilber J, Mann M, Hurt E: A novel complex of membrane proteins required for formation of a spherical nucleus. EMBO J 1998, I 7:6449-64.

FI000 Factor 6

Evaluated by Christopher Loewen 17 Jan 2012

19. Orij R, Brul S, Smits G]: Intracellular $\mathbf{p H}$ is a tightly controlled signal in yeast. Biochim Biophys Acta 20II, I 81 0:933-44.

20. Serrano R, Kielland-Brandt MC, Fink GR: Yeast plasma membrane ATPase is essential for growth and has homology with $(\mathrm{Na}++\mathrm{K}+)$, K+- and Ca2+-ATPases. Nature 1986, 3 19:689-93.

FI000 Factor 6

Evaluated by Christopher Loewen 17 Jan 2012
21. Martinez-Munoz GA, Kane PM: Vacuolar and plasma membrane proton pumps collaborate to achieve cytosolic $\mathrm{pH}$ homeostasis in yeast. J Biol Chem 2008, 283:20309-19.

FI000 Factor 6

Evaluated by Christopher Loewen 17 Jan 2012

22. Young BP, Shin JJ, Orij R, Chao JT, Li SC, Guan XL, Khong A, Jan E, Wenk MR, Prinz WA, Smits GJ, Loewen CJ: Phosphatidic acid is a pH biosensor that links membrane biogenesis to metabolism. Science 2010, 329:1085-8.

23. Kooijman EE, Burger KN: Biophysics and function of phosphatidic acid: A molecular perspective. Biochim Biophys Acta 2009, I 79 I:88I-8.

FI000 Factor 8

Evaluated by Christopher Loewen 17 Jan 2012

24. Kooijman EE, Tieleman DP, Testerink C, Munnik T, Rijkers DT, Burger KN, de Kruijff B: An electrostatic/hydrogen bond switch as the basis for the specific interaction of phosphatidic acid with proteins. J Biol Chem 2007, 282: I I356-64.

FI000 Factor 8

Evaluated by Christopher Loewen 17 Jan 2012

25. Dechant R, Peter $M$ : The $\mathbf{N}$-terminal domain of the V-ATPase subunit ' $\mathrm{a}$ ' is regulated by $\mathrm{pH}$ in vitro and in vivo. Channels (Austin) 201 I, 5:4-8.

FI000 Factor 8

Evaluated by Christopher Loewen 17 Jan 2012

26. Dechant R, Binda M, Lee SS, Pelet S, Winderickx J, Peter M: Cytosolic $\mathrm{pH}$ is a second messenger for glucose and regulates the PKA pathway through V-ATPase. EMBO J 2010, 29:25I5-26.

FI000 Factor 8

Evaluated by Christopher Loewen 17 Jan 2012

27. Reue K, Dwyer JR: Lipin proteins and metabolic homeostasis. J Lipid Res 2009, 50(Suppl):SI09-14.

28. Peterson TR, Sengupta SS, Harris TE, Carmack AE, Kang SA, Balderas E, Guertin DA, Madden KL, Carpenter AE, Finck BN, Sabatini DM: mTOR complex I regulates lipin I localization to control the SREBP pathway. Cell 20 I I, I 46:408-20.

FI000 Factor 6

Evaluated by Christopher Loewen 17 Jan 2012

29. Fang Y, Vilella-Bach M, Bachmann R, Flanigan A, Chen J: Phosphatidic acid-mediated mitogenic activation of $m$ TOR signaling. Science 200I, 294: 1942-5.

FI000 Factor 6

Evaluated by Thomas Neufeld 18 Jan 2002 